\title{
Traumatology
}

\section{Reliability, Concurrent Validity, and Cutoff Score of PTSD Checklist (PCL-5) for the Diagnostic and Statistical Manual of Mental Disorders, Fifth Edition Among Malaysian Adolescents}

Siti Raudzah Ghazali and Yoke Yong Chen

Online First Publication, April 12, 2018. http://dx.doi.org/10.1037/trm0000156

CITATION

Ghazali, S. R., \& Chen, Y. Y. (2018, April 12). Reliability, Concurrent Validity, and Cutoff Score of PTSD Checklist (PCL-5) for the Diagnostic and Statistical Manual of Mental Disorders, Fifth Edition Among Malaysian Adolescents. Traumatology. Advance online publication.

http://dx.doi.org/10.1037/trm0000156 


\title{
Reliability, Concurrent Validity, and Cutoff Score of PTSD Checklist (PCL-5) for the Diagnostic and Statistical Manual of Mental Disorders, Fifth Edition Among Malaysian Adolescents
}

\author{
Siti Raudzah Ghazali and Yoke Yong Chen \\ Universiti Malaysia Sarawak
}

\begin{abstract}
Several changes have been made to the diagnostic criteria for posttraumatic stress disorder (PTSD) in the fifth edition of the Diagnostic and Statistical Manual of Mental Disorders. The present study attempts to establish the validity and reliability of the new PTSD Checklist for the Diagnostic and Statistical Manual of Mental Disorders, Fifth Edition (PCL-5). A group of 597 Malaysian adolescents $\left(M_{\text {age }}=16.9\right.$, $S D=1.30$ ) completed the PCL-5, Harvard Trauma Questionnaire, and Patient Health Questionnaire for depression (PHQ-9). The results showed the PCL-5 has good internal consistency ( $\alpha=.91$ ), test-retest reliability $(r=.61)$, and concurrent, convergent, and discriminant validity with the Harvard Trauma Questionnaire $(r=.69)$ and the Patient Health Questionnaire for depression $(r=.56)$. Results from the receiver operating characteristic curve and kappa coefficient analysis suggest that a PCL-5 cutoff score of 33 is feasible for use among Malaysian adolescents with $72 \%$ sensitivity and $92 \%$ specificity. Thus, the PCL-5 appears psychometrically sound, and a cutoff of 33 is recommended for PTSD screening among the Malaysian adolescent population.
\end{abstract}

Keywords: assessment, trauma, youth, Malaysia, PTSD

The diagnostic criteria for posttraumatic stress disorder (PTSD) have been revised for the recent publication of the Diagnostic and Statistical Manual of Mental Disorders, Fifth Edition (DSM-5; American Psychiatric Association, 2013). Several changes were made to the diagnosis of PTSD. The criterion A2 (subjective reaction) has been eliminated (American Psychiatric Association, 2013). The avoidance and numbing symptom cluster in the Diagnostic and Statistical Manual of Mental Disorders, Fourth Edition (DSM-IV; American Psychiatric Association, 2000) has been separated into two clusters, forming four major symptom clusters in DSM-5 for PTSD: intrusion symptoms, avoidance symptoms, negative cognition and mood symptoms, and arousal and reactivity symptoms (American Psychiatric Association, 2013). DSM-5 emphasizes the concept of indirect trauma experience and the functional link between the symptoms and the traumatic events (American Psychiatric Association, 2013). In light of these changes, the present study investigates the psychometric properties of the PTSD Checklist for the Diagnostic and Statistical Manual of Mental Disorders, Fifth Edition (PCL-5) based on DSM-5 criteria among Malaysian adolescents.

Siti Raudzah Ghazali and Yoke Yong Chen, Department of Psychological Medicine, Faculty of Medicine and Health Sciences, Universiti Malaysia Sarawak.

Funding for this study was provided to Siti Raudzah Ghazali by the Malaysian Ministry of Higher Education Grant FRGS/SS02/(01)/1144/ 2014 (11).

Correspondence concerning this article should be addressed to Siti Raudzah Ghazali, Department of Psychological Medicine, Faculty of Medicine and Health Sciences, Universiti Malaysia Sarawak, Kota Samarahan 94300, Sarawak, Malaysia. E-mail: gsraudzah@fmhs.unimas.my
The PTSD Checklist (PCL) is one of the most widely used self-report assessments for PTSD (Weathers, Litz, Herman, Huska, $\&$ Keane, 1993). Previous versions have shown excellent psychometric properties with a variety of samples in different settings with test-retest reliability ranging from .75 to .96 and internal consistency from .83 to .97 (Adkins, Weathers, McDevitt-Murphy, \& Daniels, 2008; Booth-Kewley, Larson, Highfill-McRoy, Garland, \& Gaskin, 2010; Carter-Visscher et al., 2010; Weathers et al., 1993). The convergent validity of PCL was ranging from .65 to .93 (Adkins et al., 2008; Carter-Visscher et al., 2010; Weathers et al., 1993) and discriminant validity was ranging from .12 to .59 (Adkins et al., 2008; Carter-Visscher et al., 2010).

Regardless of the good psychometric properties found in PCL, even minor changes in a self-report questionnaire such as different question wording, format or even arrangement might result in changes of the measurement (Schwarz, 1999). As such, the significant changes to PTSD diagnosis from DSM-IV to DSM-5 warrant fresh examination of the psychometric properties of the PCL-5.

Evidence for the internal consistency and reliability of the PCL-5 are encouraging yet limited in scope. The PCL-5 exhibited excellent internal consistency $(\alpha=.80-.95$; Blevins, Weathers, Davis, Witte, \& Domino, 2015; Bovin et al., 2016; Demirchyan, Goenjian, \& Khachadourian, 2015; Wortmann et al., 2016), and the test reliability of the PCL-5 ranged from .82 to .86 , showing a high level of stability (Blevins et al., 2015; Bovin et al., 2016).

Psychometric evaluations of the PCL-5 have shown good construct validity, ranging from .74 to .85 (Blevins et al., 2015; Hoge, Riviere, Wilk, Herrell, \& Weathers, 2014). The PCL-5 scores demonstrated good convergent validity, ranging from .74 to .87 . For example, the PCL-5 scores were associated with other versions of PCL such as the Civilian Version $(r=.87)$, the PTSD Symptom 